\title{
Hybrid fuzzy Monte Carlo technique for reliability assessment in transmission power systems
}

\author{
Bruno Canizes, João Soares, Zita Vale, H.M. Khodr
}

A B S T R A C T

This paper proposes a new methodology to reduce the probability of occurring states that cause load curtailment, while minimizing the involved costs to achieve that reduction. The methodology is supported by a hybrid method based on Fuzzy Set and Monte Carlo Simulation to catch both randomness and fuzziness of component outage parameters of transmission power system.

The novelty of this research work consists in proposing two fundamentals approaches: 1) a global steady approach which deals with building the model of a faulted transmission power system aiming at minimizing the unavailability corresponding to each faulted component in transmission power system. This, results in the minimal global cost investment for the faulted components in a system states sample of the transmission network; 2) a dynamic iterative approach that checks individually the investment's effect on the transmission network.

A case study using the Reliability Test System (RTS) 1996 IEEE 24 Buses is presented to illustrate in detail the application of the proposed methodology.

Keywords:

Failure probability reduction

Fuzzy model

Load curtailment

Monte Carlo simulation

Reliability

Transmission power systems

\section{Introduction}

In recent years considerable power system outage events happened across the world. For example, the severe blackout in the east of North America on 14th August 2003 affected eight states in the US and two provinces in Canada, resulting in 50 million people being in darkness [1-5]. On 4th November 2006, a large blackout occurred in Europe, leading to a split of the European Union for the Coordination of Transmission of Electricity (UCTE) interconnected system into three islands with different frequencies. This incident resulted in a total load curtailment of 14,540 MW across nine European countries [2,6].

These severe power outages let us realize that the singlecontingency criterion ( $\mathrm{N}-1$ principle) that has been used for many years in the power industry may not be enough to preserve a reasonable system reliability level.

Due to the present context of power industry, costumers have become more and more well-informed about electric power systems. However, they become more cost and service reliability sensitive in the new competitive market environment and operation. They understand that it is impossible to expect almost one hundred percent continuity in power supply without almost any risk of outages [7]. In this context the transmission utilities that are responsible for transmission system planning, operation and management face huge difficulties to rationalize capital expenditures based on the possible interruptions caused by rare contingencies when considered by the deterministic criteria [8]. To overcome this deficiencies a probabilistic criteria may be used [9]. Probabilistic methods are now being more widely used in power systems operation and planning to deal with a variety of uncertainties. The most basic feature of probabilistic reliability evaluation is the modeling of stochastic factors in power systems, including random outages of system components and uncertain variation of loads. Combinations of likelihoods and consequences of random outage events create the indices that represent the systems risk [10].

An important issue in this subject is the study of the effects of weather conditions on power line outages [11-17]. Statistic data indicate that about $60 \%$ of transmission line outages are caused by adverse weather or environment [18]. The outage frequency and probability of overhead power lines increase dramatically when they are exposed to adverse weather conditions.

The reliability criteria can be deterministic or probabilistic. In both cases a consistent database and an exhaustive statistical analysis of all the available information such as failure rate $(\lambda)$ and the average repair times $(r)$ of each power systems component is 
needed. On the other hand, maximizing reliability in power systems implies minimization of unserved energy and therefore of load curtailment, avoiding an important monetary lost by undelivered energy to costumers, economy damage and an inconvenient to the power systems users.

Reducing the probability of occurrence of the states in transmission network that cause load curtailment becomes necessary and extremely important. Thus, this paper proposes a new methodology that deals with this concern. The proposed method is based on statistical failure and repair data of the transmission power system components and uses fuzzy-probabilistic modeling for system component outage parameters. With statistical records and due to uncertainties in data, the fuzzy set theory may become an attractive alternative to be used. Thus, fuzzy membership functions of system components outage parameters can be developed. To catch both randomness and fuzziness of component outage parameters, a hybrid method of fuzzy set and Monte Carlo Simulation (MCS) based on the fuzzy-probabilistic models is proposed. Once obtained the system states by MCS, a network contingency analysis is performed, to identify any overloading or voltage violation in the network. This is followed by a remedial action algorithm based on optimal power flow to reschedule generations and alleviate constraint violations and, at the same time, to avoid any load curtailment if possible or, otherwise, to minimize the total load curtailment, for the states identified by the contingency analysis. For the system states that cause load curtailment, an optimization approach is applied to reduce the probability of occurrence of those states while minimizing the costs to achieve that reduction.

The paper introduces two different approaches in the analysis of the investment concerning reliability in a given transmission network. The global steady approach handles all components in all system states that cause load curtailment in a sample of system states given by MCS. The dynamic iterative approach individually checks the effect of the investment on the transmission system for the most frequent occurring states in the MCS sample causing load curtailments. This is repeated successively, considering the impact of investments already undertaken on the failure rate and repair time, and checking the new most frequent state. The process ends when the load curtailment is zero. To illustrate the application of the proposed methodology, a case study based on Reliability Test System (RTS) 1996 IEEE 24 Buses [19] is presented and discussed.

This paper is organized as follows: Section 2 presents the creation of fuzzy set models for component outage parameters. Section 3 explains the methodology proposed to reduce the probability of occurrence of the states that cause load curtailment in transmission power systems. Section 4 presents the case study and the discussion of the obtained results. Finally, in Section 5 the most relevant conclusions are duly drawn.

\section{Using fuzzy set for components outage parameters modeling}

This section presents the state-of-art of the fuzzy set applications in power systems and the membership functions used in this paper, namely the fuzzy model for repair time and failure rate parameters.

\subsection{Fuzzy set applications in faulted power systems}

The fuzzy set concept has been applied in the power system analysis and planning [14] but very little works have been done in the application of the fuzzy set theory together with Monte Carlo Simulation (MCS) method and mathematical optimization techniques to deal with reliability calculation in transmission power systems. Pioneer work on this topic can be found in [20] and [21]. In [20] a fuzzy load model has been introduced into system reliability evaluation, whereas a probability distribution of outage data is converted into a fuzzy membership function for calculating the LOLE (Loss Of Load Expectation) index in [21]. A new approach to incorporate the fuzzy load model in system reliability assessment has been presented in [22]. Reference [10] presented a method that combines fuzzy and probabilistic techniques. A fuzzy membership function with a probabilistic mean value is developed to model outage parameters, whereas a fuzzy representation for the peak load with the probability distribution for a load curve is developed to model the system load. The same reference presents a hybrid method using fuzzy sets and MCS to evaluate fuzzy membership functions of the risk indices. Reference [12] presents a fuzzy model of overhead line weather-related outages and a combined technique of the probability theory and the fuzzy set to incorporate weather effects in transmission system reliability assessment. In [23] is presented a methodology based on optimal power flow to minimize the total load curtailment, which is supported by a hybrid method based on Fuzzy Set and Monte Carlo.

\subsection{Membership functions construction}

Collecting suitable database is essential in reliability studies. The data must sufficiently be consistent to ensure the security evaluation of the system, but must also be restrictive enough to ensure that unnecessary data are not collected. The quality of data is an important factor to consider in data collection. The reliability database should have a mean of continuous updating and should be flexible enough to output reports in a variety of adequate formats [13].

Parameters such as the failure frequency and repair time, failure rate and repair rate, transition rates between multiple states, unavailability or forced outage rate (FOR) and standard deviation will characterize each outage model. These parameters are the input data for risk evaluation and can be estimated from historical failure statistics.

For the repairable model of a single component, only two parameters are needed, and the others can be calculated from them. Most data collection systems provide the failure frequency or the unavailability and repair time. Usually, the failure and repair rates are not directly collected, but can be calculated from the failure frequency and repair time [7].

When an analytical method is used, a parameter in the outage model is the mean value based on historical statistics. In order to deal with the uncertainty in statistics, sensitivity analysis must be performed. Thus, it is necessary to have a confidence range of the estimated parameter. If MCS [24] is used, a probability distribution of the parameter is considered. So, it is necessary to estimate both the mean and standard deviation for the assumed continuous distribution of the parameter or its experimental discrete distribution [7].

\subsection{Fuzzy modeling of repair time}

The repair time parameter can be directly obtained by a data collection system $[10,23]$.

Through the direct average of repair times in different outage events, a sample mean of repair times can easily be calculated by equation (1).

$\bar{r}=\frac{1}{n} \sum_{i=1}^{n} r_{i}$

Two theorems $[7,25]$ in statistics theory enable the possibility to estimate the confidence interval of the mean. Thus, from the first 
theorem a $t$-distribution $(t)$ is used to estimate the confidence interval of the expected repair time. This is expressed in equations (2)-(4).

$r_{2}=\bar{r}-t_{\alpha / 2}(n-1) \frac{s}{\sqrt{n}}$

$r_{3}=\bar{r}+t_{\alpha / 2}(n-1) \frac{s}{\sqrt{n}}$

$r_{2} \leq \mu \leq r_{3}$

The real expected repair time $(\mu)$ is located between the lower $\left(r_{2}\right)$ and higher $\left(r_{3}\right)$ bounds which are determined by sampled repair times and it has been indicated by equation (4).

A triangle membership function of repair time (hours per failure) can be easily created with the point and interval estimates $[10,23]$, as can be seen in Fig. 1 .

The membership function equals 1 for the estimated $\bar{r}$. The significant level $\alpha$ is always a small value such as $0.05[7,10,12]$ and half of $\alpha$ is located at each of the two bounds in $t$-distribution. Conceptually, the significant level is somehow similar to the fuzzy degree represented by the membership function grade since both of them reflect a subjective confidence [10].

Once obtained $\left(r_{2}, \alpha / 2\right),(\bar{r}, 1)$, and $\left(r_{3}, \alpha / 2\right)$ points, two algebraic equations in the form of $y=m \cdot x+b$ can be defined and the $\left(r_{1}, 0\right)$ and $\left(r_{4}, 0\right)$ end points in the membership function can be calculated. It can be seen that this membership function is a symmetric one. If necessary the two bounds of repair time can also be determined or modified based on the judgment of experienced maintenance personal.

\subsection{Fuzzy modeling of failure rate}

It is quite difficult to develop an accurate method for the failure frequency interval estimation. Luckily, statistics theory enables us to estimate the confidence interval of the failure rate, which can approximately be considered as an interval estimate of failure frequency, as they are numerically close in the most cases [7].

The failure rate $\lambda$ and the failure frequency $f$ are numeric with very close values for power system components, although they are conceptually different $[7,10,23,26]$. Failure frequency and failure rate, often replace each other in practical engineering calculations of power system risk evaluation $[10,23]$.

The failure frequency is calculated as average failures per year over a time period for individual components [7,10,23]. The estimated point of failure frequency can be calculated by equation (5).

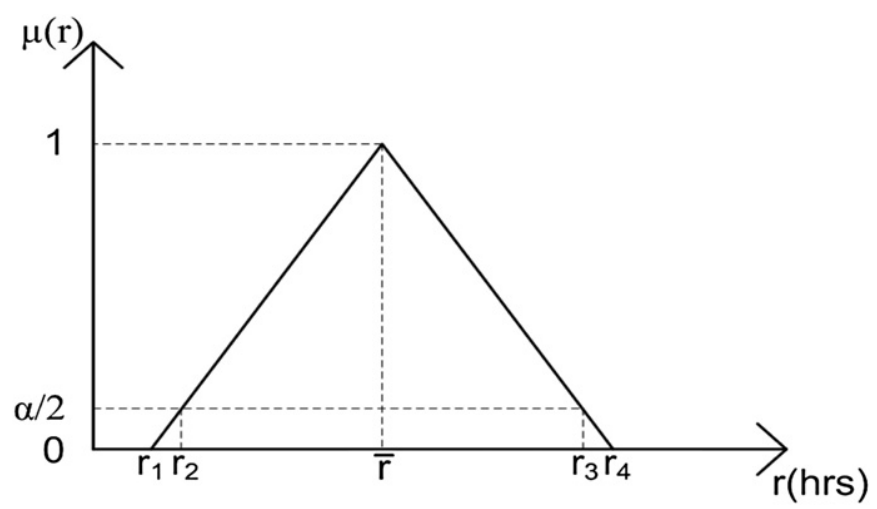

$f=\frac{R_{f}}{T}$

The absolute error of failure rate does not follow a Gaussian nor a $t$-student curve. References $[7,10,12,27]$ point in this direction and refer that in failure data processing a relationship between the ChiSquare $\left(\chi^{2}\right)$ and the Poisson distribution exists, and it may be described as:

$\chi^{2}(2 \cdot F)=2 \cdot \lambda \cdot T$

Equation (6) indicates that the quantity of two times of the expected number of failures in the time $T$ follows the $\chi^{2}$ distribution with $(2 \cdot F)$ degrees of freedom. Therefore, for a significant level $\alpha$, it can be affirmed that failure rate $\lambda$ falls into the random confidence interval (7) with the probability of $1-\alpha[7,23]$.

$\lambda_{2} \leq \lambda \leq \lambda_{3}$

$\lambda_{2}$ and $\lambda_{3}$ are expressed by equations (8) and (9)

$\lambda_{2}=\frac{\chi_{1-\alpha / 2^{(2 \cdot F)}}^{2}}{2 \cdot T}$

$\lambda_{3}=\frac{\chi_{\alpha / 2}^{2}(2 \cdot F)}{2 \cdot T}$

Using the failure rate point and interval estimates the triangle membership function of failure rate can easily be created as shown in Fig. 2.

Generally, the range between the estimated point and the higher bound is much larger than that distance between the estimated point and its lower bound when equations (8) and (9) are used.

\subsection{Fuzzy modeling of unavailability}

Using failure rate and repair time the forced outage rate or unavailability can easily be calculated $[10,23]$ using equation (10).

$U \cong \lambda \cdot r$

Through interval calculations rules for a given membership function grade, it is possible to obtain the membership function of unavailability using the failure rate and repair time membership function $[10,23]$.

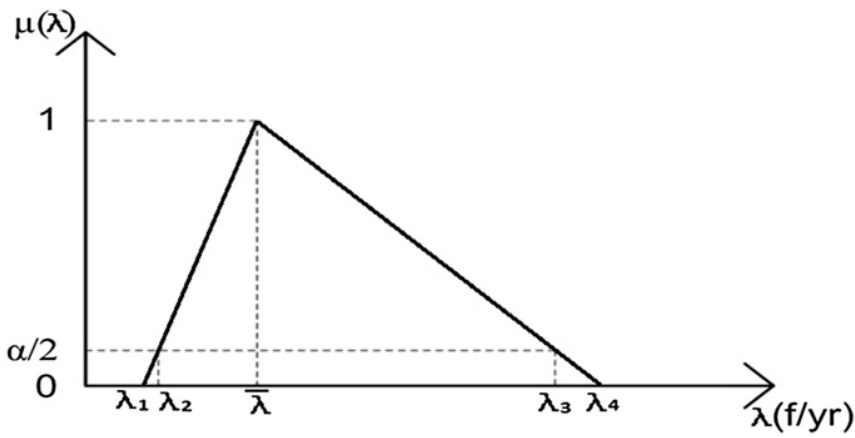

Fig. 2. Failure rate membership function $[10,23]$

Fig. 1. Repair time membership function $[10,23]$. 
Multiplying the two triangle membership function, the membership function of unavailability is no longer a triangle, as shown in Fig. 3.

\section{Proposed methodology}

The calculation of a real option value [28], when some uncertainty factors are present, can be accomplished by means of Monte Carlo simulation methods, since they are adequate for problems of high dimensionality or with stochastic parameters. However, these methods demand a very high computational time, due to the iterative process that increases according to the accuracy required. Given the dynamism of several involved decisions, there is little time for the decision making process. In this way, it is important to accelerate the Monte Carlo simulation process without loss in the required precision. Therefore, this paper presents a new methodology for the determination of a real option value under these uncertainties, also with the objective to reduce the computational time. The methodology is based on: fuzzy numbers $[29,30]$, to represent these uncertainties; and Monte Carlo simulation to obtain a good approximation of the real option value. The fuzzy number allows dealing with the technical uncertainty as a whole, avoiding the need to sample it, as it would be the case for the triangular probability distribution; this method greatly speeds up the process of the Monte Carlo simulation. The objective of the proposed methodology is also to substantially reduce the computational time involved, facilitating the decision making process. The methodology involves: fuzzy numbers, to represent certain types of uncertainties that do not have a known stochastic process that can correctly model them; stochastic processes to represent other uncertainties; and Monte Carlo simulation to generate a good approximation of the real option value.

This paper proposes a new methodology to reduce the probability of occurrence of the states that cause load curtailment in the transmission system while minimizing all involved costs to achieve that reduction.

The main basic idea of the proposed methodology is depicted in Fig. 4. This methodology is proposed only for independent forced outages.

The method has six main aspects which are presented in more detail as follows:

\subsection{Database}

An exhaustive statistical analysis of all available historical data and a consistent database creation of all available information like repair times, number of failures, number of repairs, number of

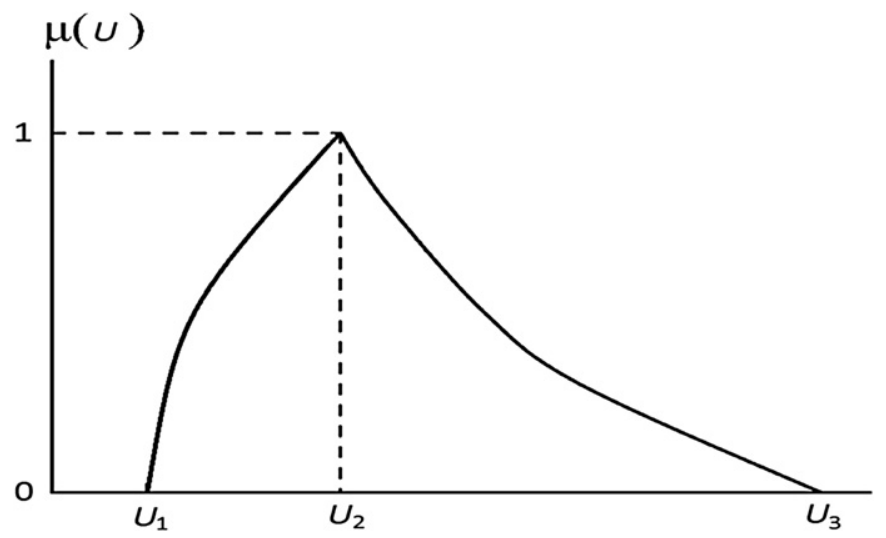

Fig. 3. Unavailability membership function $[10,23]$.

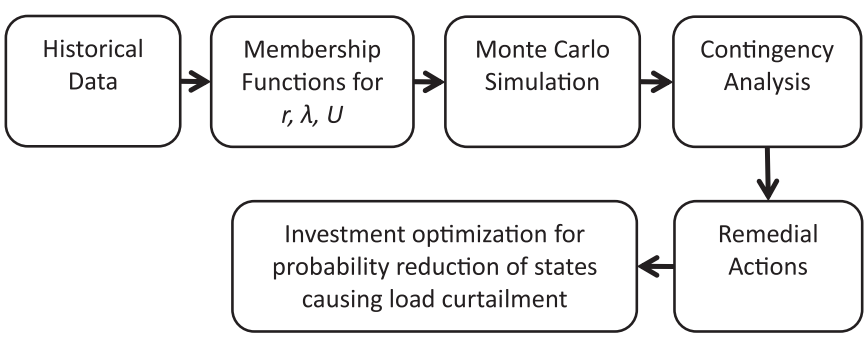

Fig. 4. Proposed methodology diagram.

repairable failures, time period analysis are the main basis for the proposed methodology.

\subsection{Repair time ( $r)$, failure rate $(\lambda)$ and unavailability $(U)$}

Weather conditions, environmental and operational conditions have an effect on transmission power systems failure frequencies or probabilities. These effect conditions are very difficult to precisely distinguish on the outage data of individual components using a probability model, since there is no (or little) statistics available. It is very common when utilities do not have sufficient statistical records of outage parameters. Thus, for all of these cases, a fuzzy approach allows to obtain adequate models.

Equations (1)-(5), and (7)-(9) allow determining the membership functions for repair times and failure rates for all system components. As a result, the membership function of unavailability can be calculated by the equation (10).

\subsection{Monte Carlo simulation}

A two-state model (up and down) is used to represent the generating units as well as the transmission system components. Transmission components include transformers, cables, overhead lines, circuit breakers, capacitors and reactors. A Nonsequential Monte Carlo Simulation [7,23,24,26,31-33] is used to obtain a sample of all possible transmission power system states.

An appropriate convergence criterion is the key to ensure accuracy in MCS. $Q$ denotes the probability of failure of at least one component in the system and $Z$ the number of system state samples. The accuracy level can be expressed by the equation (11) $[23,26]$ :

$\beta=\sqrt{\frac{1-\bar{Q}}{Z \cdot \bar{Q}}}$

\subsection{Contingency analysis}

Following one or more component failures for each failure system state, the idea is to calculate the line flows and bus voltages and to identify if there is any overloading and/or voltage constraint violation.

\subsection{Remedial actions}

When an outage causes overloading and/or voltage violation a remedial action algorithm based on optimal power flow, expressed by equations (12)-(23), is used to reschedule generation and alleviate constraint violations and, at the same time, to avoid any load curtailment if possible or, otherwise, to minimize the load curtailment for the states identified by the contingency analysis 
[23]. The minimization of the objective function (minimization of total load curtailment) is subject to all optimal power flow constraints which include the technical and logical constraints. The objective function to be minimized is:

$\min \sum_{i \in L G}$ Pcut $_{i}+$ Qcut $_{i}$

Thus, the following constraints must be considered:

Upper and lower power output limits (active and reactive power) of the generators with an integer decision variable in order to decide if the generator is working or not:

$y_{i} \cdot P G E N_{i}^{\min } \leq P G E N_{i} \leq P G E N_{i}^{\max } \cdot y_{i} \quad i \in G N$

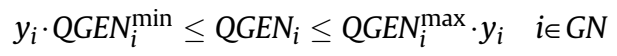

Active and reactive load curtailment limits ensuring that the load curtailment limits in bus $i$ should be lower than or equal to the load in bus $i$ :

Pcut $_{i} \leq L p_{i} \quad i \in L G$

Qcut $_{i} \leq L q_{i} \quad i \in L G$

Active and reactive power flow equations considering only the buses that have generation:

$P_{G E N}-L_{i}-P_{i}(v, \delta)+P_{c u t}=0 \quad i \in G N$

$\mathrm{QGEN}_{i}-L q_{i}-Q_{i}(\nu, \delta)+\mathrm{Qcut}_{i}=0 \quad i \in G N$

Active and reactive power flow equations considering the buses that only have loads:

$P_{i}(v, \delta)+L p_{i}-$ Pcut $_{i}=0 \quad i \in L N$

$Q_{i}(v, \delta)+L q_{i}-Q_{c u t}=0 \quad i \in L N$

Bus voltage magnitude limits:

$V_{i}^{\min } \leq V_{i} \leq V_{i}^{\max } \quad i \in N$

Bus angle limits:

$\delta_{i}^{\min } \leq \delta_{i} \leq \delta_{i}^{\max } \quad i \in N$

Capacity limits of transmission lines/cables and transmission transformers:

$S_{k}(v, \delta) \leq S_{k}^{\max }$

where, Pcut $_{i}$ and Qcut $_{i}$ are the active and reactive load curtailment at bus $i ; P G E N_{i}$ and $Q G E N_{i}$ are the generated active and reactive power at bus $i ; P G E N_{i}^{\max }$ and $P G E N_{i}^{\min }$ are the upper and lower limit of generated active power at bus $i ; Q G E N_{i}^{\max }$ and $Q G E N_{i}^{\min }$; are the upper and lower limit of generated reactive power at bus $i ; y_{i}$ is the binary variable for generator in bus $i ; L p_{i}$ and $L q_{i}$ are the active and reactive load at bus $i ; P_{i}(v, \delta)$ and $P_{i}(v, \delta)$ are the active and reactive power injections at bus $i ; V_{i}$ is the voltage magnitude at bus $i ; V_{i}^{\max }$ and $V_{i}^{\text {min }}$ are the upper and lower limit of voltage magnitude at bus $i$; $\delta_{i}$ is the voltage angle at busi; $\delta_{i}^{\max }$ and $\delta_{i}^{\min }$ are the upper and lower limit of voltage angle at bus $i ; S_{k}(v, \delta)$ is the apparent power flow on line $k$; $S_{k}^{\max }$ is the rating power limit of line $k$; GN and $L N$ are the set of generator and load buses; $L G$ is the set of buses with loads (generator bus and load bus that contain loads); $N$ is the total number of buses.

The optimal power flow model aims to minimize the total load curtailment. The states that are identified as leading to load curtailments are treated by the method described in sub-section 3.6.

\subsection{Investment optimization for probability reduction of states causing load curtailment}

The states leading to load curtailment are identified by the optimal power flow module, as described above. The corresponding components that contribute to load curtailment are also identified.

The optimization model described by equations (24)-(27) is applied in order to obtain the values of the necessary reduction for repair time $\left(\Delta r_{i}\right)$ and failure rate $\left(\Delta \lambda_{i}\right)$ of the identified components for a desired variation $\left(\Delta_{i}\right)$ in the failure probability of those components.

$$
\begin{aligned}
& \min \sum_{i=1}^{M} C_{\Delta r i} \cdot \Delta r_{i}+C_{\lambda r i} \cdot \Delta \lambda_{i} \\
& \Delta \lambda_{i}^{\min } \leq \Delta \lambda_{i} \leq \Delta \lambda_{i}^{\max } \\
& \Delta r_{i}^{\min } \leq \Delta r_{i} \leq \Delta r_{i}^{\max } \\
& \left(\lambda_{i} \cdot r_{i}\right) \cdot \Delta_{i}=\Delta \lambda_{i} \cdot r_{i}+\Delta r_{i} \cdot \lambda_{i}
\end{aligned}
$$

In order to obtain the values of $\Delta r_{i}$ and $\Delta \lambda_{i}$, the network operator must undertake analysis studies aiming to reinforce the transmission system. These studies consider the envisaged reinforcement actions (e.g. installing a line in parallel with an existing one, relocating a line, replacing a component such as a cable or a transformer) taking into account the corresponding costs. The impact of the cost of each solution in the repair time and in the failure rate of each considered component allows obtaining the values of $C_{\Delta r i}$ and $C_{\Delta \lambda i}$. Although some of the solutions may lead to non linear costs to reduce the repair and failure rate of each component, this mathematical formulation considers that these costs are linear for the considered range of repair time and failure rate reduction (i.e. in the ranges considered in equations (25) and (26)). A more sophisticated model considering non linear costs can easily be traced from the presented approach, by introducing an additional type of constraints equations (25) and (26) and the corresponding additional terms in the objective function. Binary variables should be introduced in order to guarantee that only one reinforcement solution is adopted for each component.

For this optimization model, two different approaches have been designed and implemented - the global steady and the dynamic iterative approach:

\subsubsection{Global steady approach}

The global steady approach uses the optimization model expressed by equations (24)-(27) in order to reduce the failure probability of each component in down state, particularly in the states that cause load curtailment, in a given Monte Carlo Simulation (MCS). This occurs in order to reduce the probability of occurrence of those states while minimizing the involved costs to achieve that reduction.

The flowchart of this global steady approach is shown in Fig. 5. 


\subsubsection{Dynamic iterative approach}

The dynamic iterative approach presented in Fig. 6 is based on the same optimization model. However, it uses an iterative process. Instead of optimizing all the components which are in down state, in the states that cause load curtailment, only the state that causes load curtailment with the highest probability of occurrence is optimized in each iteration. After each iteration, a new MCS is run with an updated database of the membership functions which results from the reduction obtained in the previous iteration. The stopping criteria are defined as follows:

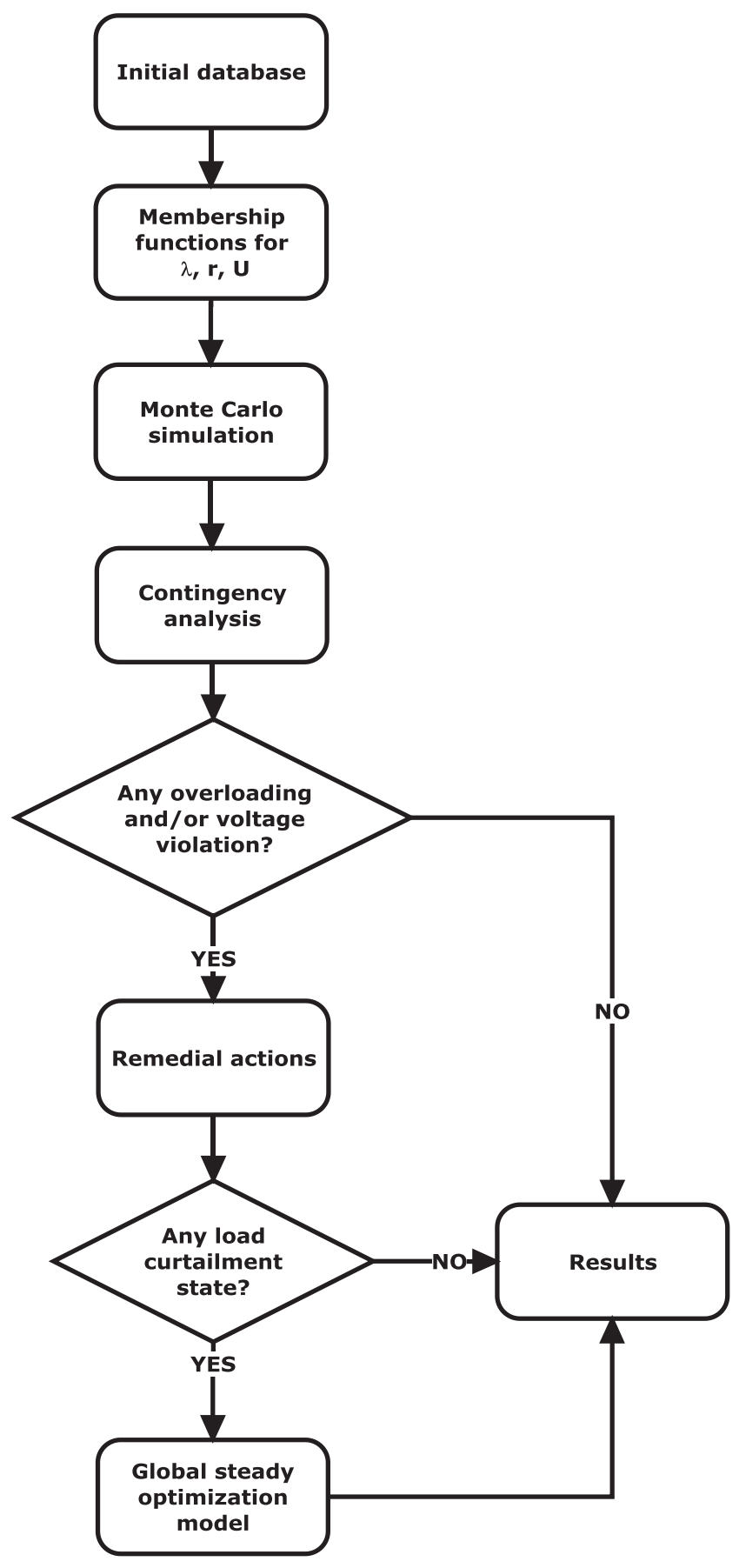

Fig. 5. Flowchart for global steady approach.

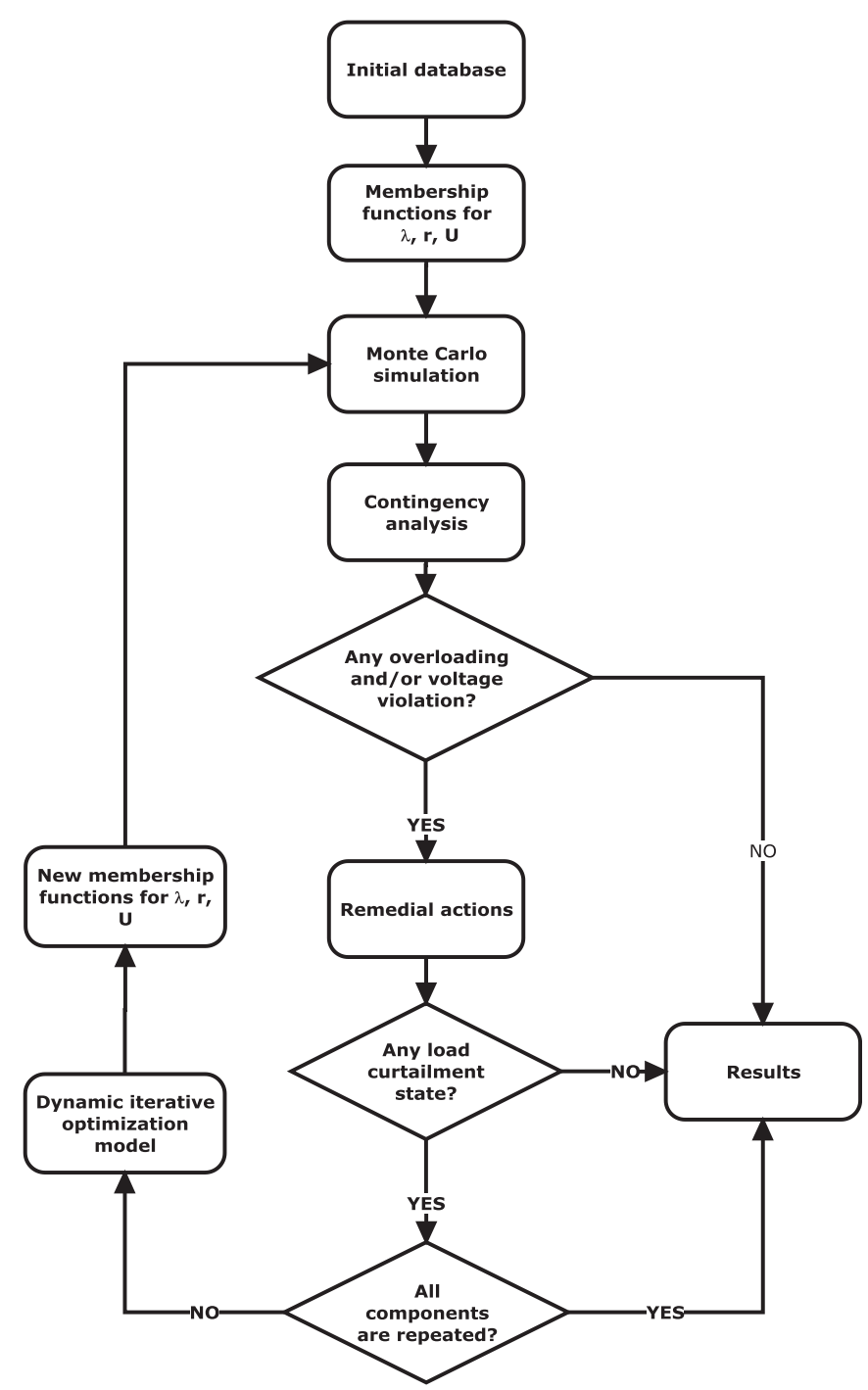

Fig. 6. Flowchart for dynamic iterative approach.

- if there are no more states with load curtailment from the last MCS run;

- only the components in the state that causes load curtailment with highest probability of occurrence are taken into account by the optimized process. If these components were already optimized in a previous iteration, then the next highest probability of occurrence of the state that causes load curtailment is selected. When there are no more states with load curtailment with new components to optimize, the algorithm is ended.

The dynamic iterative approach guarantees that there are no more load curtailment states following the stopping criteria and respecting equation (11). The global steady approach is not an iterative process, i.e. for a given MCS with a given $\beta$ the global steady approach returns the optimized components that caused load curtailment.

\section{Case study}

The proposed methodology has been tested with the Reliability Test System (RTS) 1996 IEEE 24 Buses [19] using both approaches (global steady and dynamic iterative). 
Table 1

Monte Carlo simulation results.

\begin{tabular}{|c|c|c|c|c|c|c|c|c|c|c|c|c|c|c|}
\hline \multirow{5}{*}{ Run } & \multicolumn{7}{|c|}{ Global steady approach } & \multicolumn{7}{|c|}{ Dynamic iterative approach } \\
\hline & \multicolumn{3}{|c|}{ Actual system } & \multicolumn{3}{|c|}{ Reinforced system } & \multirow{4}{*}{$\bar{Q}$ Gain } & \multicolumn{3}{|c|}{ Actual system } & \multicolumn{3}{|c|}{ Reinforced system } & \multirow[t]{4}{*}{$\bar{Q}$ Gain } \\
\hline & $\bar{Q} \mathrm{MCS} s$ & & $\beta\left(10 \mathrm{E}^{-3}\right)$ & $\bar{Q} \mathrm{MCS} s$ & & $\beta\left(10 \mathrm{E}^{-3}\right)$ & & $\bar{Q} \mathrm{MCS} s$ & & $\beta\left(10 \mathrm{E}^{-3}\right)$ & $\bar{Q}$ MCS s & & $\beta\left(10 \mathrm{E}^{-3}\right)$ & \\
\hline & \multicolumn{2}{|c|}{ Occurrences } & & \multicolumn{2}{|c|}{ Occurrences } & & & \multicolumn{2}{|c|}{ Occurrences } & & \multicolumn{2}{|c|}{ Occurrences } & & \\
\hline & No. & $\%$ & & No. & $\%$ & & & No. & $\%$ & & No. & $\%$ & & \\
\hline 1 & 318119 & 32 & 1.46 & 358513 & $36 \%$ & 1.34 & $4 \%$ & 319209 & $32 \%$ & 1.46 & 363681 & $36 \%$ & 1.32 & $4 \%$ \\
\hline 2 & 319173 & 32 & 1.46 & 357156 & $36 \%$ & 1.34 & $4 \%$ & 319303 & $32 \%$ & 1.46 & 357452 & $36 \%$ & 1.34 & $4 \%$ \\
\hline 3 & 319108 & 32 & 1.46 & 370507 & $37 \%$ & 1.30 & $5 \%$ & 318740 & $32 \%$ & 1.46 & 358384 & $36 \%$ & 1.34 & $4 \%$ \\
\hline 4 & 319001 & 32 & 1.46 & 359772 & $36 \%$ & 1.33 & $4 \%$ & 318780 & $32 \%$ & 1.46 & 356274 & $36 \%$ & 1.34 & $4 \%$ \\
\hline 5 & 318780 & 32 & 1.46 & 371332 & $37 \%$ & 1.30 & $5 \%$ & 318900 & $32 \%$ & 1.46 & 373695 & $37 \%$ & 1.29 & $5 \%$ \\
\hline 6 & 319275 & 32 & 1.46 & 358636 & $36 \%$ & 1.34 & $4 \%$ & 317616 & $32 \%$ & 1.47 & 363679 & $36 \%$ & 1.32 & $5 \%$ \\
\hline 7 & 318774 & 32 & 1.46 & 363313 & $36 \%$ & 1.32 & $4 \%$ & 318611 & $32 \%$ & 1.46 & 347320 & $35 \%$ & 1.37 & $3 \%$ \\
\hline 8 & 318258 & 32 & 1.46 & 364084 & $36 \%$ & 1.32 & $5 \%$ & 318740 & $32 \%$ & 1.46 & 358384 & $36 \%$ & 1.34 & $4 \%$ \\
\hline 9 & 317616 & 32 & 1.47 & 367955 & $37 \%$ & 1.31 & $5 \%$ & 318656 & $32 \%$ & 1.46 & 357992 & $36 \%$ & 1.34 & $4 \%$ \\
\hline 10 & 318966 & 32 & 1.46 & 353059 & $35 \%$ & 1.35 & $3 \%$ & 318900 & $32 \%$ & 1.46 & 363957 & $36 \%$ & 1.32 & $5 \%$ \\
\hline 11 & 318643 & 32 & 1.46 & 361746 & $36 \%$ & 1.33 & $4 \%$ & 319493 & $32 \%$ & 1.46 & 350832 & $35 \%$ & 1.36 & $3 \%$ \\
\hline 12 & 319303 & 32 & 1.46 & 368689 & $37 \%$ & 1.31 & $5 \%$ & 319149 & $32 \%$ & 1.46 & 350862 & $35 \%$ & 1.36 & $3 \%$ \\
\hline 13 & 318434 & 32 & 1.46 & 363709 & $36 \%$ & 1.32 & $5 \%$ & 318413 & $32 \%$ & 1.46 & 357037 & $36 \%$ & 1.34 & $4 \%$ \\
\hline 14 & 318875 & 32 & 1.46 & 366903 & $37 \%$ & 1.31 & $5 \%$ & 318656 & $32 \%$ & 1.46 & 357992 & $36 \%$ & 1.34 & $4 \%$ \\
\hline 15 & 318738 & 32 & 1.46 & 368929 & $37 \%$ & 1.31 & $5 \%$ & 318695 & $32 \%$ & 1.46 & 358579 & $36 \%$ & 1.34 & $4 \%$ \\
\hline 16 & 319004 & 32 & 1.46 & 374712 & $37 \%$ & 1.29 & $6 \%$ & 318813 & $32 \%$ & 1.46 & 355704 & $36 \%$ & 1.35 & $4 \%$ \\
\hline 17 & 319491 & 32 & 1.46 & 368289 & $37 \%$ & 1.31 & $5 \%$ & 319649 & $32 \%$ & 1.46 & 358641 & $36 \%$ & 1.34 & $4 \%$ \\
\hline 18 & 319346 & 32 & 1.46 & 368574 & $37 \%$ & 1.31 & $5 \%$ & 318997 & $32 \%$ & 1.46 & 352818 & $35 \%$ & 1.35 & $3 \%$ \\
\hline 19 & 319303 & 32 & 1.46 & 368689 & $37 \%$ & 1.31 & $5 \%$ & 318063 & $32 \%$ & 1.46 & 362492 & $36 \%$ & 1.33 & $4 \%$ \\
\hline 20 & 318119 & 32 & 1.46 & 358513 & $36 \%$ & 1.34 & $4 \%$ & 319269 & $32 \%$ & 1.46 & 358278 & $36 \%$ & 1.34 & $4 \%$ \\
\hline
\end{tabular}

The data available for the IEEE reliability test system 24 buses include the values for repair time and failure rate as well as other parameters, but does not include the historical data used to obtain them. Some adaptations in this test system were necessary to overcome the lack of essential historical data information for the proposed methodology. The following adaptations were made:

- specification of the number of reparable outages for each component of the system (lines, cables, transformers and generators) for a time period of 10 years;

- specification of the repair times for each reparable outage.

After the statistics analysis, it was found that the most adequate value for the extreme limit regarding to the objective values variations in a worth manner was 0.05 . This means that out of this limit value the objective loses coherence.

To obtain the failure states in Monte Carlo Simulation (MCS), before contingency analysis, it was necessary to define the adequate accuracy $(\beta)$ in equation (11). The authors decided that the maximum value of $\beta$ should be $1.50 E^{-03}$. After intensive running tests it was verified that $1,000,000$ cycles in MCS were enough to obtain this $\beta$ limit for this case study.

The total number of runs presented in this case study was selected as 20 for each approach (global steady and dynamic iterative) to demonstrate the accuracy of the proposed methodology. Each run involves the approaches presented in sub-section 3.5.

MATLAB language was used to create the fuzzy models for repair time, failure rate and unavailability of each system component, as well as to develop the Monte Carlo simulation algorithm used in this paper and also for contingency analysis considered in this work.

The General Algebraic Modeling System (GAMS) [34] has been used to develop the remedial action algorithm based on optimal power flow. GAMS is a high-level modeling system for mathematical programming and optimization. The solvers CPLEX and LINDOGLOBAL were used based on Mixed Integer Programming (MIP) and Non-Linear Programming (NLP) respectively.

\subsection{Numeric results}

This sub-section presents the results obtained for global steady and dynamic iterative approaches respectively. The MCS numerical results on the selected 20 runs using these methodologies are represented in Table 1 . The initial number of $\bar{Q}$ states occurred in a total of $1,000,000$ cycles is compared with the $\bar{Q}$ states after components optimization, i.e. after considering the impact of the undertaken investment. A $\bar{Q}$ MCS states corresponds to all components in up-state.

The accuracy $(\beta)$ for each run using equation (11) is presented in Table 1 . From this table one can see that the accuracy values are within the previously referred limit $\left(1.50 E^{-03}\right)$. Their variations are between $1.46 E^{-03}-1.47 E^{-03}$ and $1.29 E^{-03}-1.34 E^{-03}$ for the actual network and final network configuration respectively in global steady approach and vary between $1.46 E^{-03}-1.47 E^{-03}$ and

Table 2

Common components for all the 20 runs.

\begin{tabular}{llll}
\hline Component ID & Component & From bus & To bus \\
\hline Global steady approach & & 6 & 10 \\
10 & Transmission Cable & 7 & - \\
47 & Generator (100 MW) & 7 & - \\
49 & Generator (100 MW) & 15 & - \\
59 & Generator (155 MW) & 16 & - \\
60 & Generator (155 MW) & 18 & - \\
61 & Generator (400 MW) & 21 & - \\
62 & Generator (400 MW) & 23 & - \\
71 & Generator (350 MW) & & 10 \\
Dynamic iterative approach & 6 & - \\
10 & Transmission Cable & 7 & - \\
47 & Generator (100 MW) & 7 & - \\
48 & Generator (100 MW) & 7 & - \\
49 & Generator (100 MW) & 15 & - \\
59 & Generator (155 MW) & 16 & - \\
61 & Generator (155 MW) & 18 & - \\
62 & Generator (400 MW) & 21 & \\
70 & Generator (400 MW) & 23 & \\
71 & Generator (350 MW) & &
\end{tabular}




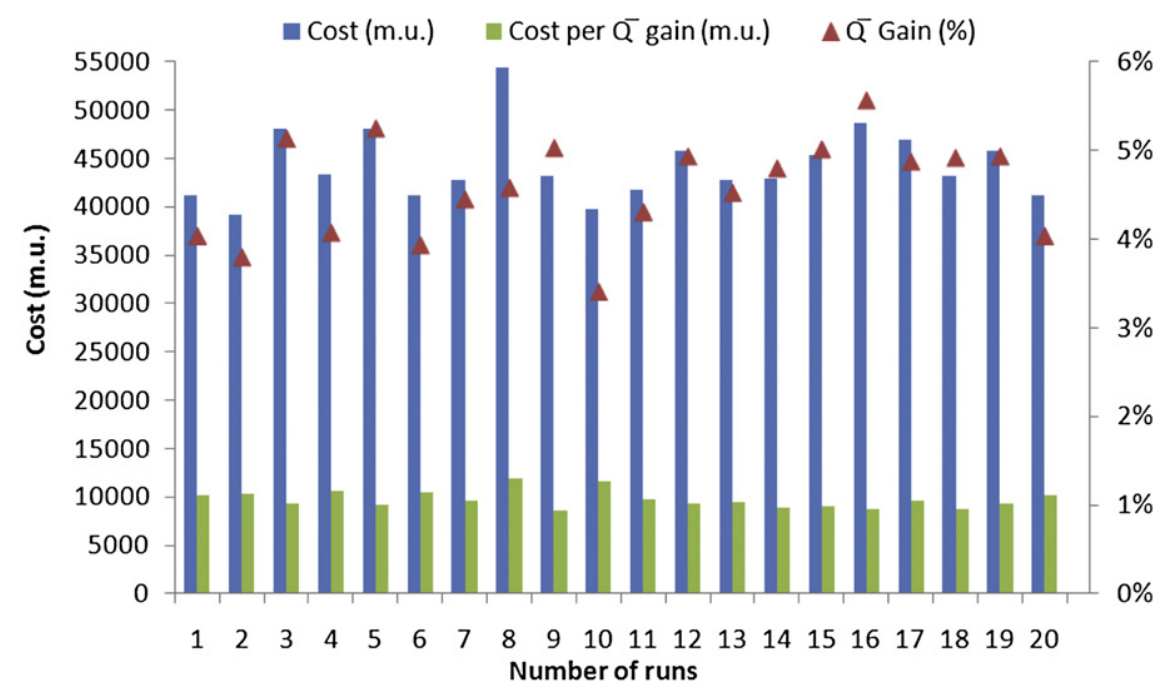

Fig. 7. Resulting costs for the global steady approach.

$1.29 E^{-03}-1.37 E^{-03}$ for the initial network and final network configuration respectively in dynamic iterative approach. Comparing both cases it can clearly be observed (Table 1) an impact in terms of reliability improvement for actual state of $5 \%$ and $4 \%$ for global steady approach and dynamic iterative approach respectively.

In order to study the sensitivity analyses of the accuracy of the model exposed in equations (24)-(27), the authors tried to investigate the influence of the variation of $\Delta_{i}$ appeared on equation (27). This variation goes from $5 \%, 10 \%, 15 \%$ and $30 \%$, but for the case of $30 \%$ the results showed a higher value in terms of investment cost corresponding to the reinforcement in transmission power systems. In this case, the benefit for the transmission power system is lower than the investment cost. On the other hand, the values of $5 \%$ and $10 \%$ are very low in terms of investment cost/benefit analysis. Thus, the authors have decided that the most adequate value in terms of investment cost/benefit is around $15 \%$.

Table 2 presents the common components which are faulted in all the states in the global steady approach and dynamic iterative approach for all 20 runs executed in this case. It is very important to notice that the unique difference between the two approaches is the component whose ID is 70. It is worthy to be mentioned that the determination of all common components (see Table 2) proves that the analysis is consistent and so are the obtained results.

Figs. 7 and 8 shows the $\bar{Q}$ gain for the 20 runs for the global steady approach and for the dynamic iterative approach respectively, which have been calculated by equation (28). For example, in the run 9 for the global steady approach (Fig. 7) the cost is 43,208 m.u. and the corresponding gain, represented on this figure by a triangle, is $5 \%$. Thus, it is obtained at 8583 m.u. cost per each $\bar{Q}$ unit gain.

Cost per $\bar{Q}$ gain $=\frac{\text { Cost }}{\bar{Q} \text { gain }} \times 0.01$

The authors criteria refer to the mean values may be adequate values to characterize the system. In the global steady approach the mean cost of all 20 runs is 44,265 m.u. whereas in the dynamic iterative approach is 40,538 m.u. The $\bar{Q}$ gain is $1 \%$ less in the dynamic iterative approach (4\%) than in the global steady approach (5\%).

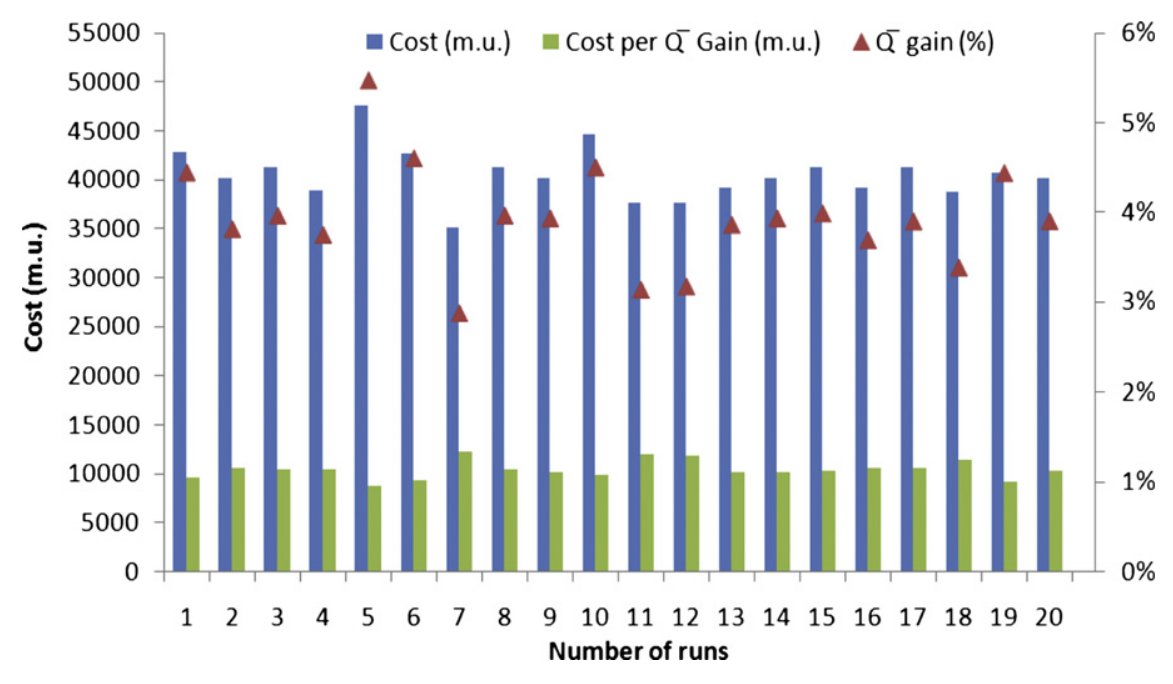

Fig. 8. Resulting costs for the dynamic iterative approach. 
The mean value cost per $\bar{Q}$ gain in the global steady approach is 9738 m.u. whereas in the dynamic iterative is 10,421 m.u.. The mean number of components optimized in the global steady approach is 17 whereas in the dynamic iterative is 14 . The mean computational execution time is 444 seconds for the global steady approach and 3048 seconds for the dynamic iterative approach.

\subsection{Results discussion}

Table 3 presents the fundamental differences in terms of the faulted components. While in the global steady approach there are 13 components that must be shifted, in the dynamic iterative approach there are only 10 components. Analyzing the presented results regarding the total cost, the global steady approach best run is run number 2 (39,188 m.u.) while for the dynamic iterative approach the best is run number 7 (35,168 m.u.). The difference between both approaches is 4020 m.u. (10.26\%).

In the case of run 7, the $\bar{Q}$ gain is $4 \%$ and $3 \%$ for global steady and dynamic iterative approach respectively (see Table 1 ). The cost per $\bar{Q}$ gain (equation (28)) is 10,317 m.u. and 12,249 m.u. for global steady and dynamic iterative approach respectively.

Tables 4 and 5 present the values of $\Delta r_{i}$ and $\Delta \lambda_{i}$ and their obtained costs for the best trial in the global steady and the dynamic iterative approach respectively.

One can conclude that the dynamic iterative approach leads to the best solution with the less total cost, since the number of optimized components in this case is lower than for the global steady approach. This fact may happen due to the dynamic iterative approach that optimizes the components of the load curtailment state with the highest probability of occurrence, whereas the global steady approach considers all the components in the load curtailment states resulting from the MCS first trial. The dynamic iterative approach, after the optimization of that load curtailment state, updates the database and checks for another load curtailment state until one of the stopping criteria is satisfied, as mentioned before. In the dynamic iterative approach the database is updated after each iteration, which causes the MCS successive runs to consider the effects of the investments already chosen in previous iterations.

Table 3

Optimized components.

\begin{tabular}{|c|c|c|c|}
\hline Component ID & Component & From bus & To bus \\
\hline \multicolumn{4}{|c|}{ Global steady approach } \\
\hline 10 & Transmission Cable & 6 & 10 \\
\hline 39 & Generator (20 MW) & 1 & - \\
\hline 44 & Generator (20 MW) & 2 & - \\
\hline 47 & Generator (100 MW) & 7 & - \\
\hline 48 & Generator (100 MW) & 7 & - \\
\hline 49 & Generator (100 MW) & 7 & - \\
\hline 59 & Generator (155 MW) & 15 & - \\
\hline 60 & Generator (155 MW) & 16 & - \\
\hline 61 & Generator (400 MW) & 18 & - \\
\hline 62 & Generator (400 MW) & 21 & - \\
\hline 69 & Generator (155 MW) & 23 & - \\
\hline 70 & Generator (155 MW) & 23 & - \\
\hline 71 & Generator (350 MW) & 23 & - \\
\hline \multicolumn{4}{|c|}{ Dynamic iterative approach } \\
\hline 10 & Transmission Cable & 6 & 10 \\
\hline 39 & Generator (20 MW) & 1 & - \\
\hline 47 & Generator (100 MW) & 7 & - \\
\hline 48 & Generator (100 MW) & 7 & - \\
\hline 49 & Generator (100 MW) & 7 & - \\
\hline 59 & Generator (155 MW) & 15 & - \\
\hline 61 & Generator (400 MW) & 18 & - \\
\hline 62 & Generator (400 MW) & 21 & - \\
\hline 70 & Generator (155 MW) & 23 & - \\
\hline 71 & Generator (350 MW) & 23 & - \\
\hline
\end{tabular}

Table 4

$\Delta r_{i}$ and $\Delta \lambda_{i}$ values and their respective costs for the global steady approach

\begin{tabular}{lllllll}
\hline Global steady (run number 2) & & & & \\
\hline Component & \multicolumn{2}{l}{$\begin{array}{l}\text { Cost applied } \\
\text { (m.u.) }\end{array}$} & $\Delta \lambda$ & $\Delta \mathrm{r}$ & \multicolumn{2}{l}{ Cost (m.u.) } \\
\cline { 2 - 6 } & $\Delta \lambda$ & $\Delta \mathrm{r}$ & & & $\Delta \lambda$ & $\Delta \mathrm{r}$ \\
\hline 10 & 3500 & 170 & 0.028 & 2.00 & 98 & 340 \\
39 & 5700 & 200 & 0.000 & 7.50 & 0 & 1500 \\
44 & 5700 & 200 & 0.000 & 7.50 & 0 & 1500 \\
47 & 6200 & 230 & 0.000 & 7.50 & 0 & 1725 \\
48 & 6200 & 230 & 0.000 & 7.50 & 0 & 1725 \\
49 & 6200 & 230 & 0.000 & 7.50 & 0 & 1725 \\
59 & 6500 & 210 & 0.000 & 6.00 & 0 & 1260 \\
60 & 6500 & 210 & 0.000 & 6.00 & 0 & 1260 \\
61 & 10,000 & 500 & 1.052 & 0.00 & 10,518 & 0 \\
62 & 10,000 & 500 & 1.052 & 0.00 & 10,518 & 0 \\
69 & 6500 & 210 & 0.000 & 6.00 & 0 & 1260 \\
70 & 6500 & 210 & 0.000 & 6.00 & 0 & 1260 \\
71 & 7500 & 300 & 0.000 & 15.00 & 0 & 4500 \\
Cost (m.u.) & & & & & 21,134 & 18,055 \\
Final Cost (m.u.) & & & & & 39,188 & \\
\hline
\end{tabular}

The process philosophy of the dynamic iterative approach makes its execution time higher. However, this is not critical in this type of planning problems.

These approaches results can help the network operator to take the adequate decisions comparing between spending 39,188 m.u. and having a $\bar{Q}$ gain of $4 \%$ or spending 35,168 m.u. and having less $1 \%$ in $\bar{Q}$ gain. The best option must apparently be the first one $(39,188$ m.u.) where the cost per $\bar{Q}$ gain is lower. However, if the network operator wants to spend less money as possible, the option of 35,168 m.u. could be suitable, as it guarantees that reinforcement actions have addressed the components with higher probability of failure that cause load curtailment.

Tables 6 and 7 show the comparison between the usage of mean values (single values) and the fuzzy membership functions. It is possible to see the considerable difference (13\%) of the number of system states without any failure when the mean values and the fuzzy membership functions are used. Thus, it is possible to conclude that many states with components in down state are not considered when the mean values are used as input data of Monte Carlo simulation. This is related to the usage of mean values leading to a particular solution of the present methodology, while with the fuzzy membership function a general solution which includes more system states with components in downstate is obtained.

Table 5

$\Delta r_{i}$ and $\Delta \lambda_{i}$ values and their respective costs for the dynamic iterative approach.

\begin{tabular}{lllllll}
\hline \multicolumn{2}{l}{ Dynamic iterative (run number 7) } & & & & \\
\hline Component & $\begin{array}{l}\text { Cost applied } \\
\text { (m.u.) }\end{array}$ & $\Delta \lambda$ & $\Delta \mathrm{r}$ & \multicolumn{2}{l}{ Cost (m.u.) } \\
\cline { 2 - 6 } & $\Delta \lambda$ & $\Delta \mathrm{r}$ & & & $\Delta \lambda$ & $\Delta \mathrm{r}$ \\
\hline 10 & 3500 & 170 & 0.028 & 2.00 & 98 & 340 \\
39 & 5700 & 200 & 0.000 & 7.50 & 0 & 1500 \\
47 & 6200 & 230 & 0.000 & 7.50 & 0 & 1725 \\
48 & 6200 & 230 & 0.000 & 7.50 & 0 & 1725 \\
49 & 6200 & 230 & 0.000 & 7.50 & 0 & 1725 \\
59 & 6500 & 210 & 0.000 & 6.00 & 0 & 1260 \\
61 & 10,000 & 500 & 1.052 & 0.00 & 10,518 & 0 \\
62 & 10,000 & 500 & 1.052 & 0.00 & 10,518 & 0 \\
70 & 6500 & 210 & 0.000 & 6.00 & 0 & 1260 \\
71 & 7500 & 300 & 0.000 & 15.00 & 0 & 4500 \\
Cost (m.u.) & & & & & 21,134 & 14,035 \\
Final Cost (m.u.) & & & & & 35,168 & \\
\hline
\end{tabular}


Table 6

Comparison between mean values and fuzzy membership function in dynamic iterative approach.

\begin{tabular}{|c|c|c|c|c|c|c|}
\hline & \multicolumn{3}{|c|}{ Mean values } & \multicolumn{3}{|c|}{ Fuzzy membership functions } \\
\hline & Cost (m.u.) & $\begin{array}{l}\text { Quantity of states } \\
\text { without any failure }\end{array}$ & $\begin{array}{l}\text { Percentage of states } \\
\text { without failures }\end{array}$ & Cost (m.u.) & $\begin{array}{l}\text { Quantity of states } \\
\text { without any failure }\end{array}$ & $\begin{array}{l}\text { Percentage of states } \\
\text { without failures }\end{array}$ \\
\hline Minimum & 30,453 & 451861 & $45 \%$ & 35,168 & 317616 & $32 \%$ \\
\hline Mean & 32,457 & 452804 & $45 \%$ & 40,538 & 318833 & $32 \%$ \\
\hline Maximum & 33,789 & 454949 & $45 \%$ & 47,618 & 319649 & $32 \%$ \\
\hline
\end{tabular}

Table 7

Comparison between mean values and fuzzy membership function in global steady approach.

\begin{tabular}{|c|c|c|c|c|c|c|}
\hline & \multicolumn{3}{|c|}{ Mean values } & \multicolumn{3}{|c|}{$\begin{array}{l}\text { Fuzzy membership } \\
\text { functions }\end{array}$} \\
\hline & Cost (m.u.) & $\begin{array}{l}\text { Quantity of states } \\
\text { without any failure }\end{array}$ & $\begin{array}{l}\text { Percentage of states } \\
\text { without failures }\end{array}$ & Cost (m.u.) & $\begin{array}{l}\text { Quantity of states } \\
\text { without any failure }\end{array}$ & $\begin{array}{l}\text { Percentage of states } \\
\text { without failures }\end{array}$ \\
\hline Minimum & 39,746 & 451311 & $45 \%$ & 39,188 & 317616 & $32 \%$ \\
\hline Mean & 44,191 & 452518 & $45 \%$ & 44,265 & 318816 & $32 \%$ \\
\hline Maximum & 53,654 & 453197 & $45 \%$ & 54,360 & 319491 & $32 \%$ \\
\hline
\end{tabular}

\section{Conclusions}

This paper presents a hybrid methodology based on Fuzzy Set and Monte Carlo Simulation to catch both randomness and fuzziness of component outage parameters that are very important to handle the uncertainty presented in this kind of problem.

A new methodology to reduce the probability of occurrence of the states that cause load curtailment while minimizing the involved costs to achieve that reduction is introduced. This is supported by an optimization model. Two alternative approaches are proposed - a global steady and a dynamic iterative one. The global approach handles all the components in all system states that cause load curtailment in a single sample of system states given by MCS. The dynamic approach uses an iterative philosophy to individually handling the state that causes load curtailment and has the highest probability of occurrence.

The main differences between the two approaches are:

- the dynamic iterative approach is more selective regarding components to optimize based on its occurrence in the successive MCS states;

- the global steady approach leads to higher costs than a dynamic one, but provides a high reinforcement of the network;

- the execution time is by far more expensive in terms of computational resources for the dynamic approach; however time is not crucial for this type of operations.

Both approaches are adequate to support the transmission system operator when planning future investments. The two alternative options resulting from each approach can be analyzed by the operator and the one that is most suited can be chosen.

\section{Acknowledgements}

This work is supported by FEDER Funds through COMPETE program and by National Funds through FCT under the projects FCOMP-01-0124-FEDER: PEst-OE/EEI/UI0760/2011, PTDC/EEA-EEL/ 099832/2008, PTDC/SEN-ENR/099844/2008, and PTDC/SEN-ENR/ $122174 / 2010$.

\section{References}

[1] Force USCPSOT. Final report on the August 14, 2003 blackout in the United States and Canada: causes and recommendations; 2004

[2] Li WY, Zhou JQ. Probabilistic reliability assessment of power system operations. Electric Power Components and Systems 2008;36(10):1102-14.

[3] LaCommare $\mathrm{KH}$, Eto JH. Cost of power interruptions to electricity consumers in the United States (US). Energy 2006;31(12):1845-55.

[4] Khazali AH, Kalantar M, Khazali A. Fuzzy multi-objective reactive power clearing considering reactive compensation sources. Energy 2011;36(5): 3319-27.

[5] Blumsack S, Fernandez A. Ready or not, here comes the smart grid! Energy 2012;37(1):61-8

[6] UCTE. Further facts about the system disturbance on 4.11.06; 2006.

[7] Li W. Risk assessment of power systems: models, methods, and applications. Hoboken, N.J; [Great Britain]: Wiley; 2005.

[8] Chowdhury AA, Koval DO. Quantitative transmission-system-reliability assessment. IEEE Transactions on Industry Applications 2010;46(1):304-12.

[9] Rei AM, Schilling MT. Reliability assessment of the Brazilian power system using enumeration and Monte Carlo. IEEE Transactions on Power Systems $2008 ; 23(3): 1480-7$

[10] Li WY, Zhou JQ Xie KG, Xiong XF. Power system risk assessment using a hybrid method of fuzzy set and Monte Carlo simulation. IEEE Transactions on Power Systems 2008;23(2):336-43.

[11] Balijepalli N, Venkata SS, Richter CW, Christie RD, Longo VJ. Distribution system reliability assessment due to lightning storms. IEEE Transactions on Power Delivery 2005;20(3):2153-9.

[12] Li W, Xiong X, Zhou J. Incorporating fuzzy weather-related outages in transmission system reliability assessment. IET Generation Transmission and Distribution 2009;3(1):26-37.

[13] Zhou YJ, Pahwa A, Yang SS. Modeling weather-related failures of overhead distribution lines. IEEE Transactions on Power Systems 2006;21(4):1683-90.

[14] Billinton R, Singh G. Application of adverse and extreme adverse weather: modelling in transmission and distribution system reliability evaluation. IEE Proceedings-Generation Transmission and Distribution 2006;153(1):115-20.

[15] Bollen MHJ. Effects of adverse weather and aging on power system reliability. IEEE Transactions on Industry Applications 2001;37(2):452-7.

[16] Bhuiyan MR, Allan RN. Inclusion of weather effects in composite system reliability evaluation using sequential simulation. IEE Proceedings-Generation Transmission and Distribution 1994;141(6):575-84.

[17] Billinton R, Li WY. A Novel method for incorporating weather effects in composite system adequacy evaluation. IEEE Transactions on Power Systems 1991:6(3):1154-60.

[18] Association CE. 2005 forced outage performance of transmission equipment equipment reliability information system; 2007.

[19] Grigg C, Wong P, Albrecht P, Allan R, Bhavaraju M, Billinton R, et al. The IEEE reliability test system - 1996. IEEE Transactions on Power Systems 1999; 14(3):1010-8

[20] Saraiva JT, Miranda V, Pinto LMVG. Generation/transmission power system reliability evaluation by Monte-Carlo simulation assuming a fuzzy load description. IEEE Transactions on Power Systems 1996;11(2):690-5.

[21] Kim JO, Singh C. Including uncertainty in LOLE calculation using fuzzy set theory. IEEE Transactions on Power Systems 2002;17(1):19-25.

[22] Li WY, Zhou JQ Lu JP, Yan W. Incorporating a combined fuzzy and probabilistic load model in power system reliability assessment. IEEE Transactions on Power Systems 2007:22(3):1386-8. 
[23] Canizes B, Vale ZA, Soares JP, Khodr HM. Fuzzy Monte Carlo mathematical model for load curtailment minimization in transmission power systems. 17th Power Systems Computation Conference (PSCC'11), Stockholm, Sweden; 2011.

[24] Fang Z. Computer simulation and Monte Carlo method. Publishing House of Beijing Industrial Institute; 1988.

[25] Freund JE. pp. xiii. Mathematical statistics, vol. 390. Englewood Cliffs: Prentice-Hall; 1962.

[26] Billinton R, Li W. Reliability assessment of electric power systems using Monte Carlo methods. New York; London: Plenum Press; 1994

[27] Mann NR, Schafer RE, Singpurwalla ND. Methods for statistical analysis of reliability and life data. New York: Wiley; 1974

[28] Zadeh LA. Fuzzy sets. Information and Control 1965;8(3):338-53.

[29] Zadeh LA. Fuzzy sets as a basis for a theory of possibility. Fuzzy Set and Systems 1999;100:9-34.

[30] Dubois D, Prade H. On several representations of uncertain body of evidence In: Gupta MM, Sanchez E, editors. Fuzzy information and decision process; 1982. p. $167-82$.

[31] Rubinstein RY. Simulation and the Monte Carlo method. New York; Chichester: Wiley; 1981

[32] Zangiabadi M, Feuillet R, Lesani H, Hadj-Said N, Kvaloy JT. Assessing the performance and benefits of customer distributed generation developers under uncertainties. Energy 2011;36(3):1703-12.

[33] Mabel MC, Raj RE, Fernandez E. Adequacy evaluation of wind power generation systems. Energy 2010;35(12):5217-22.

[34] GAMS, http://www.gams.com [accessed in April 2012].

\section{Nomenclature}

Pcut $_{i}$ : Active load curtailment at bus $i$ in p.u.

Qcut $_{i}$ : Reactive load curtailment at bus $i$ in p.u.

$P_{C E N}$ : Generated active power at bus $i$ in p.u.

$P G E N_{i}^{\max }$ : Upper limit of generated active power at bus $i$ in p.u.

$P G E N_{i}^{\text {min }}$ : Lower limit of generated active power at busi in p.u.

$P G E N_{i}$ : Generated reactive power at bus $i$ in p.u.

QGEN $N_{i}^{\max }$ : Upper limit of generated reactive power at bus $i$ in p.u.

$Q_{G E N} N_{i}^{\min }$ : Lower limit of generated reactive power at bus $i$ in p.u. $y_{i}$ : Binary variable for generator in bus $i$

$L p_{i}$ : Active load at bus $i$ in p.u.

$L q_{i}$ : Reactive load at bus $i$ in p.u.

$P_{i}(v, \delta)$ : Active power injections at bus $i$ in p.u.

$Q_{i}(v, \delta)$ : Reactive power injections at bus $i$ in p.u.

$V_{i}$ : Voltage magnitude at bus $i$ in p.u.

$V_{i}^{\max }$ : Upper limit of voltage magnitude at bus $i$ in p.u.

$V_{i}^{\text {min }}$ : Lower limit of voltage magnitude at bus $i$ in p.u.

$\delta_{i}$ : Voltage angle at bus $i$ in p.u.

$\delta_{i}^{\max }$ : Upper limit of voltage angle at bus $i$ in p.u.

$\delta_{i}^{\min }$ : Lower limit of voltage angle at bus $i$ in p.u.

$S_{k}(v, \delta)$ : Apparent power flow on line $i$ in p.u.

$S_{k}^{\max }$ : Rating power limit of line $i$ in p.u.

$L G$ : Set of buses with loads (generator bus and load bus that contain loads)

$G N$ : Set of generator buses

$L N$ : Set of load buses

$N$ : Total number of buses

$M$ : Number of failure components

$C_{\Delta r i}$ : Necessary cost to reduce the repair time of component $i$ in m.u.

$C_{\Delta \lambda i}$ : Necessary cost to reduce the failure rate of component $i$ in m.u.

$\Delta r_{i}$ : Necessary reduction of repair time variable of component $i$ in hours/failure

$\Delta \lambda_{i}$ : Necessary reduction of failure rate variable of component $i$ in failures/year

$\Delta r_{i}^{\text {min }}$ : Lower limit of necessary reduction of repair time of component $i$ in hours/ failure

$\Delta r_{i}^{\max }$ : Upper limit of necessary reduction of repair time of component $i$ in hours/ failure

$\Delta \lambda_{i}^{\mathrm{min}}$ : Lower limit of necessary reduction of failure rate of componenti in failures/ year

$\Delta \lambda_{i}^{\max }$ : Upper limit of necessary reduction of failure rate of component iin failures/ year

$\lambda_{i}$ : Fuzzy failure rate of component $i$ in failures/year (obtained by fuzzy models creation)

$r_{i}$ : Fuzzy repair time of component $i$ in hours/failure (obtained by fuzzy models creation)

$\Delta_{i}$ : Variation applied in order to reduce the unavailability of component $i$

$\bar{Q}$ : Probability of all components in up state 